\title{
Channel model of pinhole gamma camera
}

\author{
DAI QiuSheng \\ Suzhou Institute of Biomedical Engineering and Technology, Chinese Academy of Sciences, Suzhou 215163, China
}

Received February 28, 2011; accepted May 31, 2011

\begin{abstract}
The pinhole gamma camera is the basis of the newly developed method of high-resolution pinhole single photon emission computed tomography (SPECT). In this paper, a pinhole gamma camera system was examined using the channel model of information theory. The model pinhole gamma camera was simplified from a noisy, lossy channel to a noiseless, lossless channel by assuming a plane source. An approximate formula of average mutual information for the pinhole gamma camera was then derived from the model. Imaging experiments validated the applicability of the analytic formula. The findings demonstrated that the pinhole gamma camera can be adequately described using the channel model of information theory, and that average mutual information can be considered a figure of merit for the optimizing design of the pinhole gamma camera.
\end{abstract}

pinhole gamma camera, channel model, average mutual information

Citation: Dai Q S. Channel model of pinhole gamma camera. Chinese Sci Bull, 2011, 56: 2758-2763, doi: 10.1007/s11434-011-4606-9

High-resolution pinhole single photon emission computed tomography (SPECT) imaging plays an important role in the rapidly growing field of molecular imaging [1,2]. A variety of high-performance pinhole SPECT imaging systems have been constructed [3-6], but the design theory of these imaging systems is developing relatively slowly. In the design of pinhole SPECT systems, the performance of imaging systems can be predicted with simulation methods [7-11]. These methods can be used to quantify the spatial resolution, sensitivity and field of view (FOV) of the imaging systems [10-12]. These characteristics are, however, limited by each other. The improvement of any two parameters always occurs at the cost of a third parameter, so it is difficult to identify the optimal tradeoff. Therefore, it is valuable to use only one figure of merit in evaluating the overall performance of the pinhole SPECT system.

According to general information theory, the imaging process is a process of information transmission, since its main purpose is to obtain information. According to a communication model (Figure 1), an imaging object can be regarded as an information source, while the observer can

email: qiushengdai@gmail.com be regarded as a receiver. As such, the imaging system can be regarded as a channel, containing the function of an encoder. An image reconstruction algorithm is thus equivalent to a decoder. Many imaging studies simply improve the decoding capability of already existing algorithms, which does not increase the amount of information transferred by the imaging system [13-16]. If we wish to obtain more information from an information source, however, it is necessary to optimize the design of the imaging system from the perspective of the channel.

Figure 2 shows a simplified version of the channel model shown in Figure 1. This version was used for convenience the current study, which used the channel model and associated theory to examine a gamma photon imaging system. In this simplified model, the imaging object is still an information source, the imaging system is a channel, the computer receiving projection data can be regarded as the receiver, and the projection data constitute the elements in receiver space. No image reconstruction algorithm was included in the simplified communication model, to avoid any potential effects on the performance evaluation of the imaging system.

In the channel model, we directly used the average mutual information to represent the information transmission 


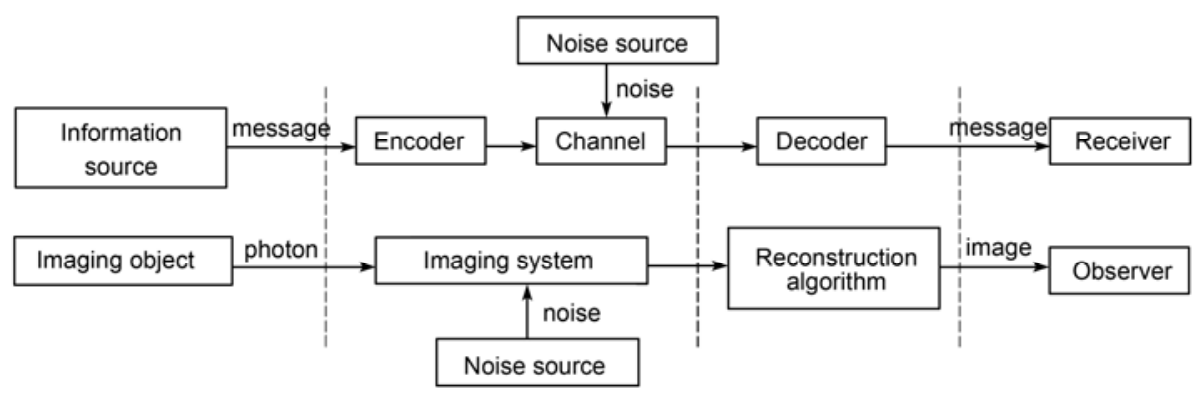

Figure 1 Schematic diagram of a communication model for photon imaging.

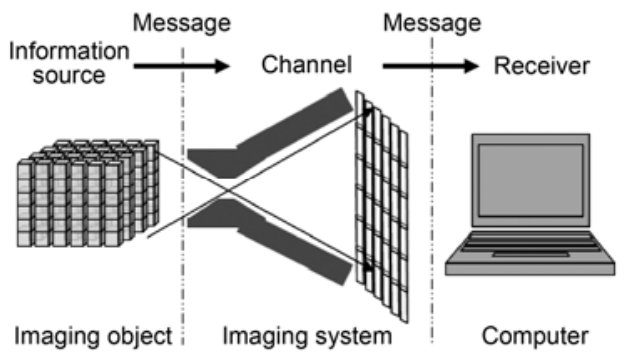

Figure 2 Schematic diagram of a communication model for a pinhole imaging system.

performance of a gamma photon imaging system. The channel capacity is related to the statistical characteristics of the channel, but the channel can achieve the greatest amount of information transmission only when it is used for a particular information source. Since the information source of a gamma photon imaging system cannot be changed freely, the channel capacity cannot be used to accurately characterize the information transmission capacity of the imaging system.

The pinhole gamma camera is the basis of the newly developed pinhole SPECT system. As the SPECT system itself is more complicated than the gamma camera, this initial investigation examines the pinhole gamma camera to establish the theoretical foundation for future studies of the pinhole SPECT system. The schematic geometry of pinhole imaging is shown in Figure 3. The spatial resolution, $R_{\text {pin }}$, and geometric efficiency, $g_{\text {pin }}$, can be approximated by eqs. (1) and (2) [17]:

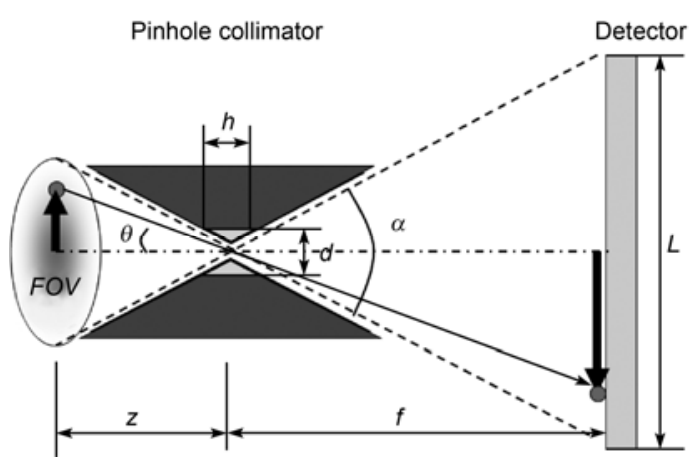

Figure 3 Schematic geometry of pinhole imaging.

$$
\begin{gathered}
R_{\text {pin }}=\sqrt{d_{\mathrm{eff}}^{2}(1+1 / M)^{2}+\left(R_{\mathrm{in}} / M\right)^{2}}, \\
g_{\text {pin }}=d_{\mathrm{eff}}^{2} \cos ^{3} \theta /\left(16 z^{2}\right),
\end{gathered}
$$

where $d_{\text {eff }}=d+\frac{\mathrm{e}^{-\mu h}}{\mu} \tan \frac{\alpha}{2}$ is the effective diameter of pinhole collimator [18], $d$ is the geometric diameter, $h$ is the channel height, $a$ is the opening angle, $\mu$ is the attenuation coefficient, $M=f / z$ is the magnification, $z$ is the source-tocollimator distance, $f$ is the focal length, $R_{\text {in }}$ is the intrinsic resolution of detector, and $L$ is the size of detector. The formula applies to the pinhole axis region.

In this paper, the approximate formula of average mutual information for the pinhole gamma camera was first derived, followed by discussion of its implications. Finally, the results of imaging experiments are reported.

\section{Channel model of pinhole gamma camera}

In this section, the channel type of pinhole gamma camera is first analyzed from the perspective of mapping, then described with a mathematical model. In this imaging model, the imaging object is divided into a $3 \mathrm{D}$ voxel matrix, and the projection image into a $2 \mathrm{D}$ pixel matrix. In $3 \mathrm{D}$ imaging space, the same radiation distribution can be mapped onto different projection images at different times due to statistical fluctuation, Compton scattering and background radiation. Thus, the pinhole SPECT system constitutes a noisy channel. For the same reasons, a variety of distributions may be mapped onto the same projection image. Thus, the pinhole SPECT system also constitutes a lossy channel. To summarize, the gamma camera can be considered a noisy and lossy channel.

According to information theory, the following mathematical language can be used to describe the channel model of the imaging system.

Let

$$
\left[\begin{array}{l}
X \\
P
\end{array}\right]=\left[\begin{array}{cccc}
X_{1}, & X_{2} & \cdots & X_{i} \cdots \\
p\left(X_{1}\right), p\left(X_{2}\right) & \cdots & p\left(X_{\mathrm{i}}\right) \cdots
\end{array}\right]
$$

be the probability space of input signal set of channel, 
where $X_{i}$ denotes the $i$ th radiation distribution, $p\left(X_{i}\right)$ is the corresponding probability, and $\sum p\left(X_{i}\right)=1$.

Let

$$
\left[\begin{array}{l}
Y \\
P
\end{array}\right]=\left[\begin{array}{ccccc}
Y_{1}, & Y_{2} & \cdots & Y_{j} & \cdots \\
p\left(Y_{1}\right), p\left(Y_{2}\right) & \cdots & p\left(Y_{j}\right) \cdots
\end{array}\right]
$$

be the probability space of the output signal set of the channel, where $Y_{j}$ denotes the $j$ th projection, $p\left(Y_{j}\right)$ is the corresponding probability, and $\sum p\left(Y_{j}\right)=1$.

Hence, the probability space of the channel is $[X, P(Y \mid X)$, $Y]$, and $\sum_{Y} P(Y \mid X)=1$. The average mutual information can be calculated by

$$
I(X ; Y)=H(X)+H(Y)-H(X Y) .
$$

In principle, all of the radioactive distributions and their corresponding projection images could be enumerated to calculate the average mutual information. However, under the conditions of current computing, there is not enough space to store all the elements of source space and receiver space. Therefore, it is impossible to calculate the average mutual information of all kinds of channels.

To overcome this difficulty, the problem was simplified by assuming a plane source. Let $R_{\text {pin }}$ be the spatial resolution of the imaging system, $D_{F O V}$ be the FOV, and $R_{\text {in }}$ be the intrinsic resolution of crystal array detector. The plane source of $D_{F O V} \times D_{F O V}$ can then be divided into a two-dimensional point source matrix model of $n_{F O V} \times n_{F O V}$ according to the resolution of the imaging system, where $n_{F O V}=D_{F O V} / R_{\text {pin }}$, the distance between points, is $R_{\text {pin }}$. Hence the number of point sources inside the FOV is

$$
n_{A}=\frac{\pi D_{F O V}^{2}}{4 R_{\mathrm{pin}}^{2}} .
$$

Eq. (1) and $D_{F O V}=L / M$ are substituted into eq. (6) resulting in

$$
n_{A}=\frac{\pi L^{2}}{4\left[d_{\mathrm{eff}}^{2}(M+1)^{2}+R_{\mathrm{in}}^{2}\right]} .
$$

In actual imaging, the concentration of radioactive material that organisms absorb has a maximum value. Here, we let $\rho_{\max }$ represent the maximum concentration of plane source, which is at the $i$ th point source. Thus, the maximum activity of the point source matrix from plane source is

$$
a_{\max }=\rho_{\max } R_{\mathrm{pin}}^{2} .
$$

In the imaging system, the count of corresponding detector units through geometric mapping is

$$
N_{i}=\xi a_{\max } g_{i} t
$$

where $\xi$ refers to the efficiency of the detector, $t$ represents measurement time, and $g_{i}$ represents the efficiency of the corresponding detector pixel:

$$
g_{i}=\left\{\begin{array}{lr}
\frac{R_{\mathrm{in}}^{2} \cos ^{3} \theta_{i}}{4 \pi(z+f)^{2}}, & d \geqslant \frac{\sqrt{2} R_{\mathrm{in}}}{1+M} \\
\frac{S_{\mathrm{mid}} \cos ^{3} \theta_{i}}{4 \pi(z+f)^{2}}, & \frac{R_{\text {in }}}{1+M} \leqslant d \leqslant \frac{\sqrt{2} R_{\mathrm{in}}}{1+M}, \\
\frac{d^{2} \cos ^{3} \theta_{i}}{16 z^{2}}, & d<\frac{R_{\mathrm{in}}}{1+M}
\end{array}\right.
$$

where

$$
\begin{aligned}
S_{\text {mid }}= & {\left[\frac{\pi}{4}-\arccos \frac{R_{\mathrm{in}}}{(1+M) d}\right](1+M)^{2} d^{2} } \\
& +R_{\mathrm{in}} \sqrt{(1+M)^{2} d^{2}-R_{\mathrm{in}}^{2}} .
\end{aligned}
$$

The gamma photons passing through the pinhole aperture to the detector are mainly divided into three components: direct, penetration and scattering. In general, the direct component is regarded as signal, and penetration, scattering and background radiation are regarded as noise. As such, geometric aperture was used in eq. (10) rather than effective aperture. The information transmission efficiency of the collimator is given by

$$
\eta=\gamma_{d} /\left(\gamma_{d}+\gamma_{p}+\gamma_{s}+\gamma_{b}\right)
$$

where $\gamma_{d}$ is the direct component, $\gamma_{p}$ is the penetration component, $\gamma_{s}$ is the scattering component, and $\gamma_{b}$ is the background radiation component.

While $N_{i} \gg>1$, the standard deviation of $N_{i}, \sigma$, can be approximated by $\sqrt{N_{i}}$, here $2 \sigma$ was used as a unit of gray scale. Considering the effect of noise on the information transmission capacity of the imaging system, $\eta$ was used directly to calibrate the effective gray scale. Thus, the effective gray scale of projection image is given by

$$
K_{i}=\eta\left(\frac{N_{i}}{2 \sqrt{N_{i}}}+1\right)=\eta\left(\frac{\sqrt{\xi a_{\max } g_{i} t}}{2}+1\right)
$$

Therefore, one gray image approximately corresponds to only one plane source, and the pinhole gamma camera approximates the noiseless, lossless channel. Therefore, when $a_{\max }$ is at the $i$ th point source, the number of elements in receiver space is $K_{i}^{n_{A}-1}$, and the mutual information is

$$
I_{i}=\left(n_{A}-1\right) \log _{2} K_{i} \text {. }
$$

Since $i \in\left[1, n_{A}\right]$, the average mutual information of the imaging system is

$$
\begin{aligned}
I & =\frac{1}{n_{A}} \sum_{i=1}^{n_{A}} I_{i}=\frac{n_{A}-1}{n_{A}} \sum_{i=1}^{n_{A}} \log _{2} K_{i} \\
& =\frac{n_{A}-1}{n_{A}} \sum_{i=1}^{n_{A}} \log _{2} \eta\left(\frac{\sqrt{\xi a_{\max } g_{i} t}}{2}+1\right) .
\end{aligned}
$$


When $n_{A}>1$,

$$
I \approx \sum_{i=1}^{n_{A}} \log _{2} \eta\left(\frac{\sqrt{\xi a_{\max } g_{i} t}}{2}+1\right)
$$

According to eq. (15), an increase of $\xi a_{\max } t$, causes the average mutual information to increase. This indicates that the gamma photon imaging system is a time-dependent channel; thus, the receiver space in the model above is not complete. If the value of eq. (15) is less than 0 , the artifacts produced by noise would lead the image to produce an error message. The average mutual information is generally not less than 0 in information theory, so in the following calculation, it is defined as $I=0$, when $I<0$.

Comparing eq. (2) and (10) shows that the channel model increases the amount of information about the geometric relationship between the pinhole and the detector unit. When $d_{\text {eff }} \geqslant \sqrt{2} R_{\text {in }} /(1+M)$, further increasing the aperture can increase the sensitivity of conventional parameters, but cannot increase the count of corresponding detector units through point source geometric mapping. On the other hand, it would be expected to decrease the average mutual information in the system because of the reduction in $n_{A}$. Therefore, an optimal aperture should exist for the actual imaging system. Similarly, according to eq. (10), an optimal focal length should exist for the same imaging object or the same FOV. From (15), the optimal aperture and optimal focal length can be obtained under different parameters. The opening angle of the pinhole collimator is determined by the focal length and detector size.

\section{Analysis results}

In accord with eq. (15), the effects of FOV, focal length, aperture and intrinsic resolution on the average mutual information were initially examined. The calculation model was as follows: the pinhole collimators made of tungsten alloy were knife-edge-shaped, the area of the $\mathrm{NaI}(\mathrm{Tl})$ crystal array detector was $120 \mathrm{~mm} \times 120 \mathrm{~mm}$, and the radioactive source was ${ }^{99 \mathrm{~m}} \mathrm{Tc}, 140.5 \mathrm{keV}$ radiation. In the calculation, $\eta=\gamma_{d} /\left(\gamma_{d}+\gamma_{p}+\gamma_{s}+\gamma_{b}\right) \approx\left(d / d_{\text {eff }}\right)^{2}$, the values of $\xi \rho_{\max } t$ were $106 / \mathrm{mm}^{2}$ and $10^{7} / \mathrm{mm}^{2}$ respectively.

Figure 4 shows the relationship between the average mutual information and the focal length under different magnifications, and different conditions of FOV. The magnifications tested were 1, 2, 3 and 4 respectively, the pinhole aperture was $1.0 \mathrm{~mm}$, the intrinsic resolution of the detector was $2.0 \mathrm{~mm}, \xi \rho_{\max } t$ was $10^{6} / \mathrm{mm}^{2}$ in Figure $4(\mathrm{a})$, and $10^{7}$ $/ \mathrm{mm}^{2}$ in Figure 4(b). As shown in the figure, an optimal focal length existed, but this increased with the magnitude of the $\xi \rho_{\max } t$ increase. In addition, FOV truncation caused substantial information loss when the imaging object was too big.

Figure 5 shows the relationship between the average mutual
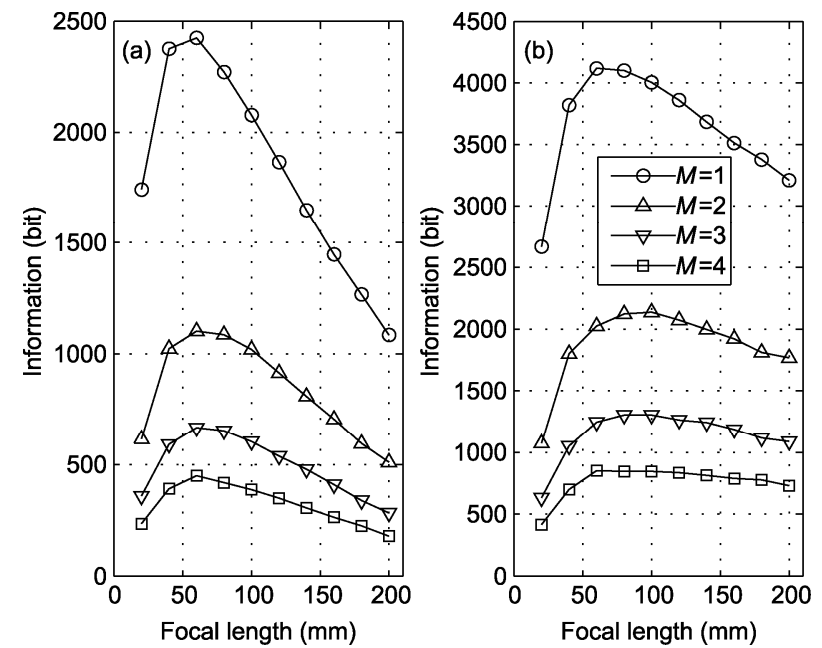

Figure 4 Relationship between the average mutual information and the focal length. (a) $\xi \rho_{\max } t=10^{6} / \mathrm{mm}^{2}$; (b) $\xi \rho_{\max } t=10^{7} / \mathrm{mm}^{2}$.
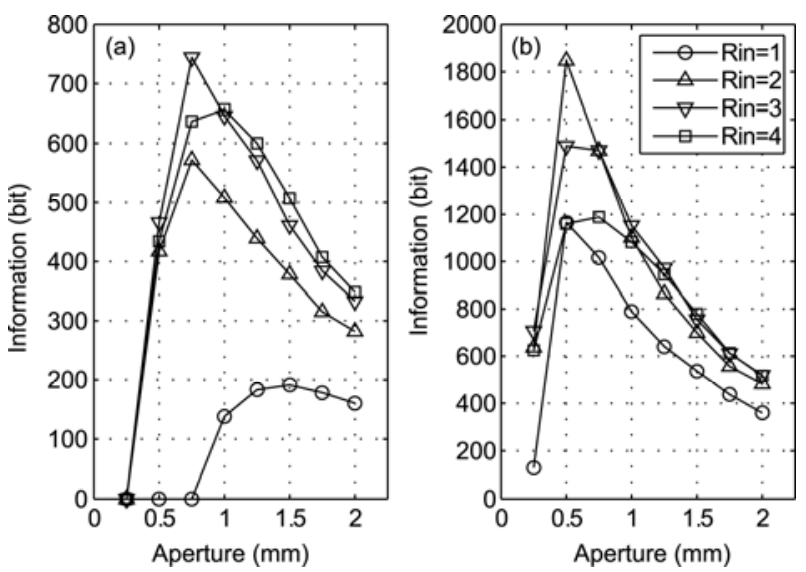

Figure 5 Relationship between the average mutual information and the aperture. (a) $\xi \rho_{\max } t=10^{6} / \mathrm{mm}^{2}$; (b) $\xi \rho_{\max } t=10^{7} / \mathrm{mm}^{2}$;

information and the aperture under different intrinsic resolutions. The intrinsic resolutions tested were 1, 2, 3 and $4 \mathrm{~mm}$, while the focal length was $100 \mathrm{~mm}$, the source-to-collimator distance was $30 \mathrm{~mm}, \xi \rho_{\max } t$ was $10^{6} / \mathrm{mm}^{2}$ in Figure 5(a), and $10^{7} / \mathrm{mm}^{2}$ in Figure 5(b). As shown in the figure, an optimal aperture existed, but it was related to the intrinsic resolution and the count detected by the detector.

The above analysis indicates that an optimal focal length, aperture, and intrinsic resolution of pinhole gamma camera exist in practical applications of this imaging method, which seems to conflict with eq. (1). According to eq. (1), the smaller the aperture and intrinsic resolution, the higher the spatial resolution, and the more information can be obtained from the image. In fact, eq. (1) describes the spatial resolution of the pinhole gamma camera under the ideal state, not taking into account the effects of statistical fluctuation, transmission, scattering or background radiation noise. As shown in Figure 5, increasing the statistical count (i.e. de- 
creasing the statistical noise), caused a gradual decrease in the optimal aperture and the intrinsic resolution. In practical imaging applications, the statistical count is limited and is not too big due to the constraints imposed by radioactive drugs and imaging time. Thus, optimal values of aperture and intrinsic resolution exist, but vary under different conditions of use.

\section{Imaging results}

To verify the conclusions described above, a series of pinhole gamma camera imaging experiments were designed. The imaging object consisted of four capillaries, with an internal diameter of $0.3 \mathrm{~mm}$, filled with approximately 20 $\mathrm{mCi} / \mathrm{mL}{ }^{99 \mathrm{~m}} \mathrm{Tc}$ solution. The distances between capillaries were $1 \mathrm{~mm}, 3 \mathrm{~mm}$ and $2 \mathrm{~mm}$ respectively (Figure 6). Imaging experiments were completed with a small gamma camera in the MicroSPECT/CT laboratory of the Shanghai Institute of Applied Physics (SINAP), CAS. The gamma camera consisted of a $\mathrm{NaI}(\mathrm{Tl})$ crystal array and a positionsensitive photo-multiplier tube (PSPMT) with crystal thickness of $5 \mathrm{~mm}$ and intrinsic resolution of $1.4 \mathrm{~mm}$. The focal length was $10 \mathrm{~cm}$. The pinhole collimators were made of tungsten alloy, with apertures of 0.5 and $1.0 \mathrm{~mm}$, channel height of $0.5 \mathrm{~mm}$, and acceptance angle of 60 degrees.

We first calculated the analysis values with different object distances and different acquisition times (Figure 7), in which the object distances were 20, 40, 60, 80 and $100 \mathrm{~mm}$, respectively, the acquisition times were 30 and $300 \mathrm{~s}$, respectively, $a_{\mathrm{max}}=\rho \pi r^{2} R_{\mathrm{pin}}$. As the FOV varied at different object distances, the information density was used for comparison, which is the average mutual information per unit area.

Projection images were then obtained at different object distances and different acquisition times. The profiles of projection images are shown in Figure 8.

As shown in Figure 8(a) and (c), a $0.5 \mathrm{~mm}$ aperture could distinguish a space interval of $1 \mathrm{~mm}$ when $L=20 \mathrm{~mm}$

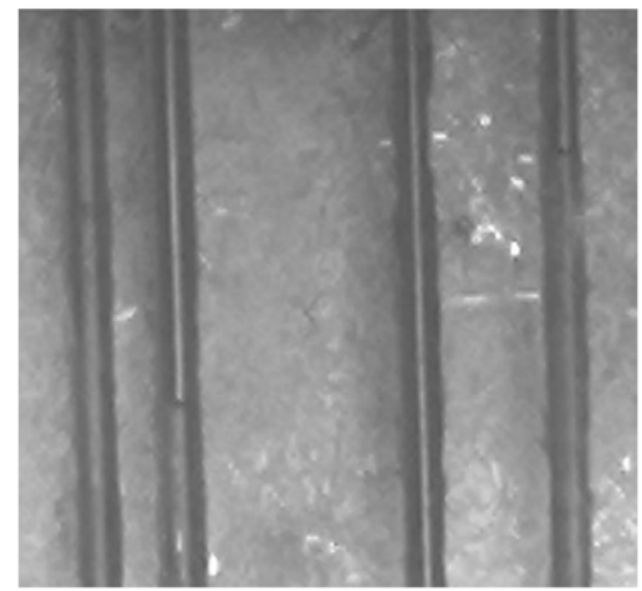

Figure 6 Imaging object consisting of four capillaries.

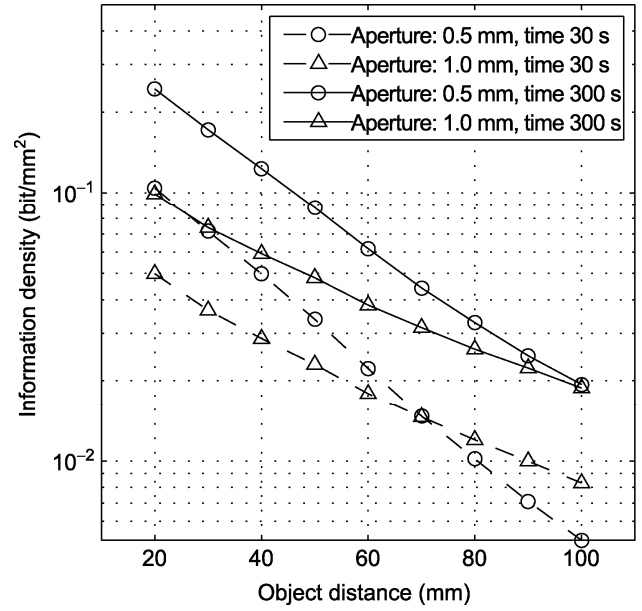

Figure 7 Analysis values of average mutual information.
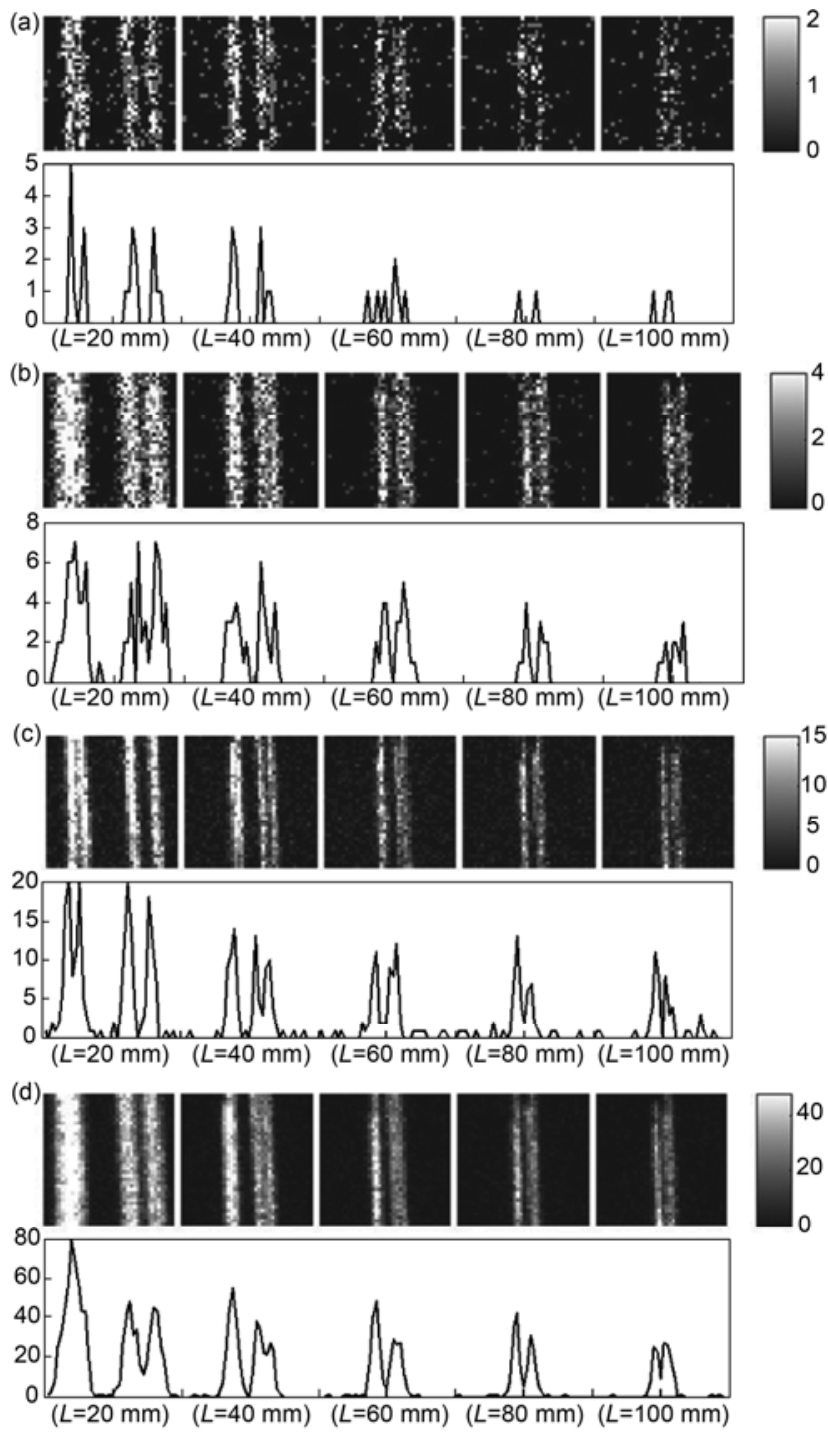

Figure 8 Projection images of capillary and profiles with different apertures, different times and different object distances. (a) The aperture is $0.5 \mathrm{~mm}$, acquisition time is $30 \mathrm{~s}$; (b) the aperture is $1.0 \mathrm{~mm}$, acquisition time is $30 \mathrm{~s}$; (c) the aperture is $0.5 \mathrm{~mm}$, acquisition time is $300 \mathrm{~s}$; (d) the aperture is 1.0 $\mathrm{mm}$, acquisition time is $300 \mathrm{~s}$. 
and acquisition time is $300 \mathrm{~s}$, indicating that the amount of information increases with an increase in acquisition time. From Figure 8(a) and (b), the signal to noise ratio (SNR) of the $0.5 \mathrm{~mm}$ aperture decayed more quickly with increasing object distance compared to when the aperture was $1.0 \mathrm{~mm}$. When $L=80 \mathrm{~mm}$, a $0.5 \mathrm{~mm}$ aperture could not distinguish a $3 \mathrm{~mm}$ space interval, whereas an $1.0 \mathrm{~mm}$ aperture could. This finding indicates that an $1.0 \mathrm{~mm}$ aperture is better than a $0.5 \mathrm{~mm}$ aperture under these imaging conditions. Comparing Figure 7 and Figure 8 indicates that the experimental results were consistent with the theoretical predictions.

The results described above indicate that the proposed formula was able to predict the experiment results, and that an optimal aperture exists in this imaging system under different imaging conditions. Therefore, an optimized design of pinhole gamma camera system should be based on the characteristics of the imaging object, including its size, the desired imaging time, and the concentration and specificity of radioactive substances.

\section{Conclusion}

Although further studies are required, the current results revealed that the channel model of information theory is appropriate for describing the pinhole gamma camera. In addition, the findings indicated that average mutual information can replace resolution, sensitivity and FOV in providing descriptions of the properties of imaging systems. The formula used to approximate the pinhole gamma camera presented here was able to determine optimized parameters under different imaging conditions, providing valuable information for optimal design and use. Future studies will examine the channel model of the pinhole SPECT imaging system on the basis of this theory. In addition, the current method can be used for optimizing the design of other imaging systems, such as photon counting computed tomography.

The author would like to thank Yujin Qi of the Shanghai Institute of Applied Physics for the experiment instruments.
1 Meikle S R, Kench P, Kassiou M, et al. Small animal SPECT and its place in the matrix of molecular imaging technologies. Phys Med Biol, 2005, 50: R45-R61

2 Madsen M T. Recent advances in SPECT imaging. J Nucl Med, 2007, 48: 661-673

3 Schramm N U, Ebel G, Engeland U, et al. High-resolution SPECT using multipinhole collimation, IEEE Trans Nucl Sci, 2003, 50: 315-320

4 Furenlid L R, Wilson D W, Chen Y C, et al. Fast SPECT II: A second-generation high-resolution dynamic SPECT imager. IEEE Trans Nucl Sci, 2004, 51: 631-635

5 Beekman F J, van der Have F, Vastenhouw B, et al. U-SPECT-I: A novel system for submillimeter-resolution tomography with radiolabeled molecules in mice. J Nucl Med, 2005, 46: 1194-1200

6 van der Have F, Vastenhouw B, Ramakers R M, et al. U-SPECT-II: An ultra-high-resolution device for molecular small-animal imaging. J Nucl Med, 2009, 50: 599-605

7 Song T Y, Choi Y, Chung Y H, et al. Optimization of pinhole collimator for small animal SPECT using Monte Carlo simulation. IEEE Trans Nucl Sci, 2003, 50: 327-332

8 Beekman F J, Vastenhouw B. Design and simulation of a high-resolution stationary SPECT system for small animals. Phys Med Biol, 2004, 49: 4579-4592

9 Cao Z, Bal G, Accorsi R, et al. Optimal number of pinholes in multi-pinhole SPECT for mouse brain imaging-a simulation study. Phys Med Biol, 2005, 50: 4609-4624

10 Rentmeester M C M, van der Have F, Beekman F J. Optimizing multi-pinhole SPECT geometries using an analytical model. Phys Med Biol, 2007, 52: 2567-2581

11 Vunckx K, Beque D, Defrise M, et al. Single and multipinhole collimator design evaluation method for small animal SPECT. IEEE Trans Med Imaging, 2008, 27: 36-46

12 Vanhove C, Defrise M, Lahoutte T, et al. Three-pinhole collimator to improve axial spatial resolution and sensitivity in pinhole SPECT. Eur J Nucl Med Mol Imaging, 2008, 35: 407-415

13 Zhang X Z, Qi Y J. Development of fully 3D image reconstruction techniques for pinhole SPECT imaging. Chinese Sci Bull, 2011, 56: 340-348

14 Yuan S, Dong Z L, Miao L, et al. Research on the reconstruction of fast and accurate AFM probe model. Chinese Sci Bull, 2010, 55: 2750-2754

15 Lu D T, Zhang T, Yang J Q, et al. A reconstruction method of porous media integrating soft data with hard data. Chinese Sci Bull, 2009, 54: 1876-1885

16 Zhao X, Lu J, Gong A D, et al. Operational spectrum reconstruction of data from the Fourier transform hyperspectral imager onboard HJ1A satellite, Chinese Sci Bull, 2010, 55: 1808-1812

17 Anger H O. Radioisotope Cameras in Instrumentation in Nuclear Medicine. New York: Academic press, 1967. 485-552

18 Dai Q S, Qi Y J. Investigation of the spatial resolution of pinhole SPECT imaging (in Chinese). Acta Phys Sin, 2010, 59: 1370-1378

Open Access This article is distributed under the terms of the Creative Commons Attribution License which permits any use, distribution, and reproduction in any medium, provided the original author(s) and source are credited. 